PROF BRIAN J ANDERSON (Orcid ID : 0000-0002-2826-3019)

DR GEORGE A CHALKIADIS (Orcid ID : 0000-0002-5613-1654)

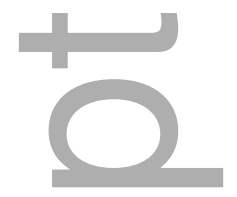

$\square$

Article type

: Editorial

\title{
Tramadol; keep calm and carry on
}

\section{Brian J Anderson $^{1}$, Jane Thomas ${ }^{2}$, Kaye Ottaway ${ }^{3}{ }_{A N D}$ George A $^{\text {Chalkiadis }}{ }^{4}$}

1. Department of Anaesthesiology, Faculty of Medicine and Health Science, University of Auckland, Park Rd, Auckland 1023, New Zealand

E-mail:briana@adhb.govt.nz

2. Department of Anaesthesia, Starship Children's Hospital, Park Rd, Auckland 1023, NZ

E-mail:. JaneThom@adhb.govt.nz

3. Department of Anaesthesiology, Faculty of Medicine and Health Science, University of Auckland, Park Rd, Auckland 1023, New Zealand

E-mail: kotter@xtra.co.nz

4. Department of Paediatric Anaesthesia and Pain Management, Royal Children's Hospital, Flemington Rd, Parkville, Victoria 3052, Australia

E-mail: George.Chalkiadis@rch.org.au

This is the author manuscript accepted for publication and has undergone full peer review but has not been through the copyediting, typesetting, pagination and proofreading process, which may lead to differences between this version and the Version of Record. Please cite this article as doi: $10.1111 /$ pan.13190

This article is protected by copyright. All rights reserved 


\section{Correspondence:}

Prof Brian Anderson, Department of Anesthesiology, Faculty of Medicine and Health Science, University of Auckland, Park Rd, Auckland 1023, New Zealand

Key Words: tramadol, opioids, tonsillectomy, obstructive sleep apnea, genetic polymorphisms

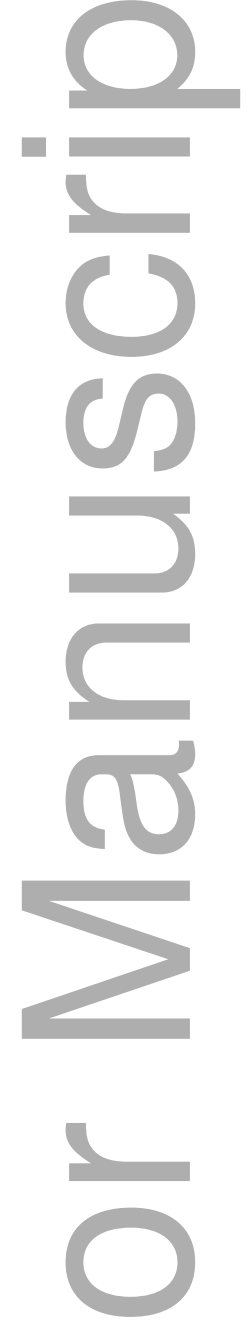

\section{Introduction}

Tramadol is a weak opioid that is structurally related to morphine and codeine.(1) Two enantiomers provide analgesia; one is an opioid $\mu$-receptor agonist, and the other inhibits neuronal reuptake of serotonin and norepinephrine, thus producing "multimodal antinociception."(1) It is widely used in children as a second-line analgesic or rescue medication when paracetamol and NSAIDs prove inadequate.

An important metabolic pathway is that provided by CYP2D6 to form $O$-desmethyltramadol; the active M1 metabolite. This active M1 metabolite has a $\mu$-receptor affinity approximately 200 times greater than tramadol.(2) CYP2D6 is the same enzyme responsible for the production of codeine's 
major active metabolite, morphine. Tramadol has come under increasing scrutiny ever since codeine was identified as a contributing factor to death in children suffering obstructive sleep apnea who underwent tonsillectomy.(3) The USA Food and Drug Administration (FDA) issued a warning in 2013 concerning the use of codeine in children after adenotonsillectomy surgery following a number of deaths that were attributed to overdoses associated with undiagnosed ultra-rapid polymorphisms of CYP2D6.

\section{FDA announcement}

Regrettably, tramadol has now been tarred with the same brush as codeine. The FDA identified nine cases of serious breathing problems, including three deaths, with the use of tramadol in children younger than 18 years from January 1969 to March 2016. The FDA recently announced (20 April 2017) that it is restricting the use of codeine and tramadol medicines in children. Their recommendations include the following:

1. The FDA's strongest warning, called a Contraindication, to be added to the drug labels of codeine and tramadol alerting that codeine should not be used to treat pain or cough and tramadol should not be used to treat pain in children younger than 12 years.

2. A new Contraindication to the tramadol label, warning against its use in children younger than 18 years to treat postoperative pain following adenotonsillectomy.

3. A new Warning to the drug labels of codeine and tramadol to recommend against their use in adolescents between 12 and 18 years who are obese or have conditions such as obstructive sleep apnea or severe lung disease, which may increase the risk of serious breathing problems

4. A strengthened Warning to mothers that breastfeeding is not recommended when taking codeine or tramadol medicines due to the risk of serious adverse reactions in breastfed infants. These can include excess sleepiness, difficulty breastfeeding, or serious breathing problems that could result in death.

Such warnings as these issued by the FDA highlight potential dangers in order to protect patients. They can be considered part of the process of drug education. These warnings are "living documents" and will change with new evidence; they are not "coffins" for drugs. Both succinylcholine $(4,5)$ and droperidol $(6,7)$ have been part of this process in the past. The use of general anaesthesia in children under the age of 2 years is currently being reviewed.(8)

\section{Tramadol is not codeine}

Tramadol is not quite the same as codeine. Both the parent drug and its M1 metabolite are active. There is also more than one clearance pathway for the parent drug. Tramadol exerts its analgesic activity via at least two complementary mechanisms: opioid activity through activating the $\mu$-opioid receptor (encoded by gene OPRM1) by the parent drug and M1 metabolite, and monoaminergic 
activity through weak inhibition of norepinephrine and serotonin reuptake by the parent drug to enhance inhibitory effects on pain transmission in the spinal cord.

Tramadol is extensively metabolized in the liver by $\mathrm{O}$ - and $\mathrm{N}$-demethylation and by conjugation reactions to form glucuronides and sulfates. Elimination of tramadol and its metabolites is predominantly through the kidneys. The O-demethylation of tramadol to its major analgesic metabolite, O-desmethyltramadol (M1), is catalyzed by CYP2D6, whilst N-demethylation to Ndesmethyltramadol (M2) is catalyzed by CYP2B6 and CYP3A4. Inactivation of O-desmethyltramadol is by glucuronidation in the liver, mostly via UGT2B7 and UGT1A8.

Polymorphisms of the CYP2D6 enzyme have long been known to contribute different responses to tramadol. Higher concentrations of the (+)-M1 metabolite and greater analgesic efficacy of tramadol has been reported in extensive metabolizers compared to poor metabolizers. There also appears to be less nausea, vomiting and fatigue amongst poor metabolizers. $(2,9)$ In addition to the mu receptor effect, the reduced uptake of serotonin in descending spinal cord pathways may also contribute to the emetic effect of tramadol.(2)

The pharmacokinetics of tramadol have been investigated from neonates to adults.(10-13) Unfortunately, the identification of genotype does not predict phenotype. For example, those classed as poor metabolizers may have normal clearance.(11) Tramadol provides analgesia both from the parent compound (target concentration $100 \mathrm{ng} / \mathrm{ml}$ ) and from its M1 metabolite (target concentration $15 \mathrm{ng} / \mathrm{ml}$ ).(14) The low incidence of respiratory depression and constipation, fewer controls on use, and similar frequency of nausea and vomiting (10\% to $40 \%$ ) compared to other opioids have made tramadol an attractive alternative to stronger opioid analgesics such as morphine and oxycodone.(12)

\section{Other analgesics metabolised by CYP2D6}

The CYP2D6 enzyme system is responsible for metabolism of other analgesic drugs that have not been restricted by the FDA. Hydrocodone is also metabolized by the CYP2D6 enzyme system to the active metabolite hydromorphone. One study of adult females after Caesarean section found that $60 \%$ were extensive metabolizers, $30 \%$ intermediate, $3 \%$ poor, and $7 \%$ ultra-rapid. This genotypic variability can have profound effects on effectiveness as well as the potential for toxicity with ultrarapid metabolism.(15) A paediatric study of African American children treated for sickle cell disease, found a similar distribution of metabolic conversion from hydrocodone to hydromorphone.(16)

Oxycodone is a long-acting semisynthetic opioid that is metabolised through CYP3A-mediated Ndemethylation to noroxycodone and CYP2D6 O-demethylation to oxymorphone and noroxymorphone. $(17,18)$ Oxymorphone accounts for $10 \%$ of the circulating oxycodone metabolites 
and is 14 times more potent than oxycodone because of its 40 -fold greater affinity for the mu-opioid receptor compared with oxycodone. Consequently concerns about "fast metabolisers" when the drug is used for obstetric pain in breast feeding mothers have been expressed. $(19,20)$ Fast metabolisers have been implicated in oxycodone related deaths in adults.(21)

\section{Tramadol toxicity}

Respiratory depression attributable to tramadol overdose is described and is associated with doses in excess of 7-10 $\mathrm{mg} / \mathrm{kg}$ in children younger than 6 years, although seizures have been reported after a minimal dose of $4.8 \mathrm{mg} / \mathrm{kg}$.(22-24) However, evidence that polymorphism of CYP2D6 contributes to reported toxicity is lacking. There is a report of one 5 year old child prescribed a single tramadol dose (formulation comprised oral drops containing $100 \mathrm{mg} / \mathrm{mL}$ ) after adenotonsillectomy. He presented the next morning with opioid intoxication and was resuscitated. Genotyping of CYP2D6 was conducted, and three functional alleles were found that were consistent with ultra-rapid metabolism. Although a single urine sample was taken for metabolite concentration, no plasma samples were available for assay and interpretation is difficult.(25)

The three fatalities reported by the FDA occurred outside the USA in children younger than 6 years. Elevated serum tramadol concentrations were noted in all three, suggesting overdose. The reasons for tramadol use in these children was postoperative pain following tonsillectomy and clubfoot surgery, and to manage fever. All three children were administered tramadol oral drops $(100 \mathrm{mg} / \mathrm{mL})$. This formulation is no longer available in New Zealand and has been replaced with a more preferable tramadol $10 \mathrm{mg} / \mathrm{mL}$ elixir.

There is no question that ultra-metabolisers of tramadol could potentially get into trouble in the following circumstances:

1. Excessive dosing - due to iatrogenic, intentional or inadvertent parental overdose.

2. Children with known respiratory disease have increased opioid sensitivity - of particular concern are those with obstructive sleep apnea secondary to tonsillar hypertrophy or obesity.(26)

One hospital has addressed the CYP2D6 polymorphism issue by identifying the CYP2D6 polymorphisms in hundreds of children undergoing adenotonsillectomy and obviating the use of codeine in those with the poor and ultra-rapid polymorphisms.(27) The rest of the children were successfully managed with codeine after tonsillectomy. A similar approach could be used for tramadol. 


\section{Broader Considerations}

The last 25-30 years have witnessed an improvement in the delivery of appropriate pain relief for children. Regrettably, a knee jerk reaction to any reported death or overdose attributable to any analgesic drug has been to either deny that drug (e.g. codeine) or reduce the dose (e.g., paracetamol) in children. A significant increase in opioid-related addiction and deaths in adults with chronic nonmalignant pain in the USA and Australia have contributed to recent recommendations to limit the use of opioids in this setting. This issue should not be confused with the use of appropriate opioid therapy in patients with acute pain and cancer-related pain.

Alternative analgesic drugs (e.g., oxycodone) may also be associated with genetic variability with clearance or contribute to community substance abuse when the prescribed medication is not used by the intended recipient. Alternatives such as oral morphine have high pharmacokinetic variability (28) and those individuals with low clearance and obstructive apnoea will also be prone to respiratory compromise. All opioids must be used wisely after any surgical procedures. Better discharge education for parents that could include observing them prepare analgesia doses prior to discharge, safer low concentration formulations, and understanding pain trajectories after common surgical procedures may be better options than banning any particular drug in children.

The prescription of opioids and tramadol in paediatric and adolescent patients with chronic nonmalignant pain is generally reserved for relatively short-term use to facilitate functional rehabilitation. There are no published trials looking at the short or long-term efficacy of opioid therapy in children with chronic non-malignant pain. With regard to tramadol, there is one case study published that reported the efficacy of tramadol for treating chronic pain associated with Ehlers-Danlos syndrome in two siblings.(29) Pharmacological management is consequently largely empirical and extrapolated from adult pain management strategies. Chronic opioid therapy may contribute to ongoing pain due to opioid-induced hyperalgesia. Furthermore, there is no reason why the well-known adverse effects of chronic opioid therapy in adults, including tolerance, dependence and addiction, immunosuppression, cognitive dysfunction and sedation, impaired bowel motility and constipation would not be expected to occur in children and adolescents. Inadvertent sibling misuse and opioid diversion are also potential problems. Hence, cautious use of tramadol and other opioid analgesics is recommended in this patient group.

The search for better analgesic drugs continues. One drug that could replace tramadol is tapentadol. It has a similar mechanism of action to tramadol but analgesic activity is independent of enzyme systems and it is has no active metabolites.(30) It has been available for adult use as an immediate and sustained release oral formulation in Australia since 2014, but it is not currently registered in NZ. 
It is available as a $20 \mathrm{mg} / \mathrm{mL}$ liquid formulation in the USA and Europe. Unfortunately, there are no paediatric efficacy or safety trials published.

\section{Recommendations}

1. Tramadol dose should be limited for acute pain after tonsillectomy (e.g., maximum dose 1 $\mathrm{mg} / \mathrm{kg}$ 6-8 h, max $400 \mathrm{mg} /$ day). Tramadol overdose is greater danger than CYP variants.

2. Children with obstructive sleep apnoea who have undergone tonsillectomy should continue to be monitored in hospital overnight to assess both response and sensitivity to opioids before discharge.

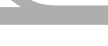

Conflicts of interest: Brian Anderson sits on the Editorial Board of the journal Pediatric Anesthesia. No other conflicts of interest are declared

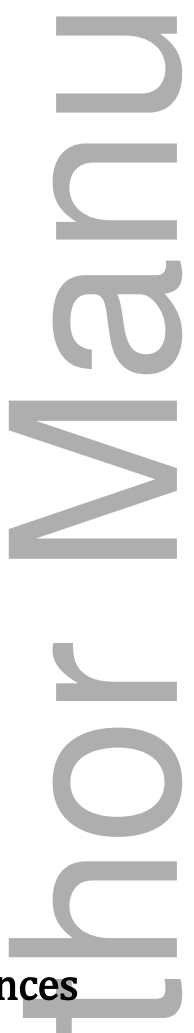

\section{References}

1 Grond S, Sablotzki A. Clinical pharmacology of tramadol. Clin Pharmacokinet 2004; 43: 879923.

2 Poulsen L, Arendt-Nielsen L, Brosen K, et al. The hypoalgesic effect of tramadol in relation to CYP2D6. Clin Pharmacol Ther 1996; 60: 636-644.

3 Tobias JD, Green TP, Cote CJ, et al. Codeine: Time to Say "No". Pediatrics 2016; 138.

4 Morell RC. FDA group urges sux label reduced to "warning". Journal of clinical monitoring 1995; 11: 141-143. 

this drug be contraindicated for routine use in children and adolescents? (Summary of the discussions of the anesthetic and life support drug advisory meeting of the Food and Drug Administration, FDA building, Rockville, MD, June 9, 1994). Anesth Analg 1995; 80: 207-208.

6 Faine B, Hogrefe C. News flash: Old Mother Hubbard reports the cupboard is bare...time for the FDA to let droperidol out of the (black) box. The Annals of pharmacotherapy 2012; 46: 12591261.

7 Matlock A, Allan N, Wills B, et al. A continuing black hole? The FDA boxed warning: an appeal to improve its clinical utility. Clinical toxicology (Philadelphia, Pa) 2011; 49: 443-447.

8 Hansen TG. Use of anaesthetics in young children: Consensus statement of the European Society of Anaesthesiology, the European Society for Paediatric Anaesthesiology, the European Association of Cardiothoracic Anaesthesiology and the European Safe Tots Anaesthesia Research Initiative. European journal of anaesthesiology 2017; 34: 327-328.

9 Enggaard TP, Poulsen L, Arendt-Nielsen L, et al. The analgesic effect of tramadol after intravenous injection in healthy volunteers in relation to CYP2D6. Anesth Analg 2006; 102: 146-150. 10 Allegaert $\mathrm{K}$, Anderson $\mathrm{BJ}$, Verbesselt $\mathrm{R}$, et al. Tramadol disposition in the very young: an attempt to assess in vivo cytochrome P-450 2D6 activity. Br J Anaesth 2005; 95: 231-239.

11 Allegaert K, Holford N, Anderson BJ, et al. Tramadol and o-desmethyl tramadol clearance maturation and disposition in humans: a pooled pharmacokinetic study. Clin Pharmacokinet 2015;

54: $167-178$

12 Allegaert K, Rochette A, Veyckemans F. Developmental pharmacology of tramadol during infancy: ontogeny, pharmacogenetics and elimination clearance. Paediatr Anaesth 2011; 21: 266273.

13 Allegaert K, van den Anker JN, de Hoon JN, et al. Covariates of tramadol disposition in the first months of life. Br J Anaesth 2008; 100: 525-532.

14 Garrido MJ, Habre W, Rombout F, et al. Population pharmacokinetic/pharmacodynamic modelling of the analgesic effects of tramadol in pediatrics. Pharm Res 2006; 23: 2014-2023.

15 Stauble ME, Moore AW, Langman L, et al. Hydrocodone in postoperative personalized pain management: pro-drug or drug? Clin Chim Acta 2014; 429: 26-29.

16 Yee MM, Josephson C, Hill CE, et al. Cytochrome P450 2D6 polymorphisms and predicted opioid metabolism in African American children with sickle cell disease. J Pediatr Hematol Oncol 2013; 35: e301-305. 

pharmacokinetic association study in pediatric surgical patients. Pharmacogenomics 2017; 18: 337348.

18 Samer CF, Daali Y, Wagner M, et al. Genetic polymorphisms and drug interactions modulating CYP2D6 and CYP3A activities have a major effect on oxycodone analgesic efficacy and safety. Br J Pharmacol 2010; 160: 919-930.

19 Lam J, Kelly L, Ciszkowski C, et al. Central nervous system depression of neonates breastfed by mothers receiving oxycodone for postpartum analgesia. J Pediatr 2012; 160: 33-37 e32.

20 Olkkola KT, Hamunen K, Seppala T, et al. Pharmacokinetics and ventilatory effects of intravenous oxycodone in postoperative children. Br J Clin Pharmacol 1994; 38: 71-76.

21 Jannetto PJ, Wong SH, Gock SB, et al. Pharmacogenomics as molecular autopsy for postmortem forensic toxicology: genotyping cytochrome P450 2D6 for oxycodone cases. J Anal Toxicol 2002; 26: 438-447.

22 Hassanian-Moghaddam H, Farnaghi F, Rahimi M. Tramadol overdose and apnea in hospitalized children, a review of 20 cases. Res Pharm Sci 2015; 10: 544-552.

23 Stassinos GL, Gonzales L, Klein-Schwartz W. Characterizing the Toxicity and Dose-Effect Profile of Tramadol Ingestions in Children. Pediatr Emerg Care 2017.

24 Spiller HA, Gorman SE, Villalobos D, et al. Prospective multicenter evaluation of tramadol exposure. J Toxicol Clin Toxicol 1997; 35: 361-364.

25 Orliaguet G, Hamza J, Couloigner V, et al. A case of respiratory depression in a child with ultrarapid CYP2D6 metabolism after tramadol. Pediatrics 2015; 135: e753-755.

26 Waters KA, McBrien F, Stewart P, et al. Effects of OSA, inhalational anesthesia, and fentanyl on the airway and ventilation of children. J Appl Physiol (1985) 2002; 92: 1987-1994.

27 Gammal RS, Crews KR, Haidar CE, et al. Pharmacogenetics for Safe Codeine Use in Sickle Cell Disease. Pediatrics 2016; 138.

28 Dawes JM, Cooke EM, Hannam JA, et al. Oral morphine dosing predictions based on single dose in healthy children undergoing surgery. Paediatr Anaesth 2016.

29 Brown SC, Stinson J. Treatment of pediatric chronic pain with tramadol hydrochloride: siblings with Ehlers-Danlos syndrome - Hypermobility type. Pain Res Manag 2004; 9: 209-211. 30 Langford RM, Knaggs R, Farquhar-Smith P, et al. Is tapentadol different from classical opioids? A review of the evidence. Br J Pain 2016; 10: 217-221.

This article is protected by copyright. All rights reserved 


\section{University Library}

\section{- M M I N E R VA A gateway to Melbourne's research publications}

Minerva Access is the Institutional Repository of The University of Melbourne

Author/s:

Anderson, BJ;Thomas, J;Ottaway, K;Chalkiadis, GA

Title:

Tramadol: keep calm and carry on.

Date:

2017-08

Citation:

Anderson, B. J., Thomas, J., Ottaway, K. \& Chalkiadis, G. A. (2017). Tramadol: keep calm and carry on.. Paediatr Anaesth, 27 (8), pp.785-788. https://doi.org/10.1111/pan. 13190.

Persistent Link:

http://hdl.handle.net/11343/293164 